\title{
Design of bionic goat quadruped robot mechanism and walking gait planning
}

\author{
Fu Zhang ${ }^{1,2,3^{*}}$, Shuai Teng ${ }^{1}$, Yafei Wang ${ }^{1}$, Zhijun Guo ${ }^{4}$, Jiajia Wang ${ }^{1}$, Ruiliang $\mathrm{Xu}^{4}$ \\ (1. College of Agricultural Equipment Engineering, Henan University of Science and Technology, Luoyang 471003, Henan, China; \\ 2. Key Laboratory of Modern Agricultural Equipment and Technology, Ministry of Education, Jiangsu University, Zhenjiang 212013, \\ Jiangsu, China; \\ 3. Collaborative Innovation Center of Machinery Equipment Advanced Manufacturing of Henan Province, Luoyang 471003, Henan, China; \\ 4. College of Vehicle \&Transportation Engineering, Henan University of Science and Technology, Luoyang 471003, Henan, China)
}

\begin{abstract}
In order to improve the walking stability and obstacle jumping ability of the robot on the slope, the goat was taken as the bionic prototype, a kind of bionic goat quadruped robot has been designed. By the bionic principle, the mechanical structure of the quadruped robot was designed, and the Denavit-Hartenberg (D-H) method was used to build the kinematic model, through which the forward and inverse kinematics of the robot was calculated, thus the equations of velocity and acceleration of the joint angle change were obtained during the quadruped robot motion, providing control theory foundation for robots. During walk gait planning, a low contact compact foot trajectory planning method using a high order polynomial curve was used to carry out the foot trajectory planning of the robot for the swing phase and stance phase. Through MATLAB software simulation, the simulation results of MATLAB software showed that the trajectory of the foot of the quadruped robot was semi-elliptic in a gait cycle, which were basically consistent with the foot trajectory obtained from the previous goat slope walking test. Therefore, the simulation results by MATLAB software showed that the foot trajectory was reasonable. Based on this, a gait plan was carried out, and the sequence of the lift leg steps of the robot was obtained. The test platform for the whole machine was built. The results showed that the height of the leg of the quadruped robot was $7.37 \mathrm{~cm}$, and the length of the gait was $28.40 \mathrm{~cm}$. Compared with the planned average gait length $S=30 \mathrm{~cm}$ and average step height $H=7 \mathrm{~cm}$, the error of the leg height and the gait length was $5.28 \%$ and $5.33 \%$, respectively. The designed gait planning achieved the expected results. The knee joint angle varied from $126.3^{\circ}$ to $156.1^{\circ}$, and the hip joint angle varied from $103.9^{\circ}$ to $138.4^{\circ}$. The changes in the joint angle of the quadruped robot could prove the correctness of the foot motion trajectory planning, which will provide a theoretical basis for the structural design and gait planning of the quadruped robot.
\end{abstract}

Keywords: bionic goat, quadruped robot, mechanism design, gait plan, D-H method, foot trajectory

DOI: $10.25165 /$ j.ijabe.20201305.5769

Citation: Zhang F, Teng S, Wang Y F, Guo Z J, Wang J J, Xu R L. Design of bionic goat quadruped robot mechanism and walking gait planning. Int J Agric \& Biol Eng, 2020; 13(5): 32-39.

\section{Introduction}

The mobile robot platform has developed into a research hotspot, and can be divided into a tracked robot, wheeled robot, footed robot and rail robot, according to its movement mode ${ }^{[1-5]}$. Different types of mobile robots can be used in special environments such as military reconnaissance, seismic rescue, detection pipelines, resource exploration, bionic walking, etc. With broad application prospects, more and more scholars are studying related technologies such as robot design, gait motion control, and environmental adaptability. Foot robots are generally divided into biped robots, quadruped robots, and hexapod robots.

\section{Received date: 2020-03-09 Accepted date: 2020-07-28}

Biographies: Shuai Teng, Master candidate, research interest: intelligent agricultural equipment and technology, Email: 285149837@qq.com; Yafei Wang, $\mathrm{PhD}$ candidate, research interest: intelligent agricultural equipment and technology, Email: 946584218@qq.com; Zhijun Guo, PhD, Professor, research interest: biomimetic technology for ground machinery, Email: gzhj1970@ 163.com; Jiajia Wang, PhD, Lecturer, research interest: biomechanics and bionic, Email: johnnyjiajia@163.com; Ruiliang Xu, Associate Professor, research interest: modern mechanism design method and application, Email: lyxrl@163.com.

*Corresponding author: Fu Zhang, PhD, Professor, Assistant Dean, research interest: the biomimetic technology and the intelligent agricultural equipment. College of Equipment Agricultural Engineering, Henan University of Science and Technology, Luoyang 471003, China. Tel: +86-13592065683, Email: zhangfu30@126.com.
Huang et al. ${ }^{[6]}$ proposed a method that can determine the smooth motion of the hip joint of the most significant stability margin by using two parameters. The motion track of the hip joint was obtained through iterative calculation, and the walking mode of the biped robot was planned. Zhang et al. ${ }^{[7]}$ and Yin et al. ${ }^{[8]}$ improved the motion continuity and stability of the quadruped robot through reasonable planning of its gait. Faigl et al. ${ }^{[9]}$ designed an adaptive motion controller applied to a hexapod robot, which can accurately detect the contact points between the legs and the ground, so that the hexapod robot can walk on rough terrain.

Tracked robots have better passability on irregular roads ${ }^{[10,11]}$, but they have a larger volume and weight and are less flexible. Wheeled robots move faster ${ }^{[12,13]}$, but they have a weaker ability to overcome obstacles. Footed robots have good obstacle surmounting ability and kinematic flebility ${ }^{[14]}$. Quadruped robots not only have the stability of a biped robot, but they also avoid the redundancy and complexity of hexapod robots. Quadruped robots have strong adaptability to unstructured environments due to its discrete support method and have both the motion characteristics of wheeled and tracked robots ${ }^{[15-19]}$. In quadruped robots, two main sources of power include hydraulic and motor-driven power, such as the Japanese four-legged hydraulically-driven walking robot TITAN XI, which can walk on slopes as smoothly as on the level ground $^{[20]}$. Gao et al. ${ }^{[21]}$ proposed a new decoupling control method that used mechanical-hydraulic coupling as the coupling behavior of a hydraulic foot robot. This method reduced the 
impact of system coupling and achieved coordinated motion between multiple joints. Compared with the hydraulic drive, the motor drive is advantageous for energy transfer, where the signal can be received quickly, and the response sensitivity is high. Jiao et al. ${ }^{[22]}$ designed a tracked robot driven by a DC motor. The robot based on adaptive sliding mode control has good control accuracy and can meet the needs of the operation. Zhao et al. ${ }^{[23]}$ developed an electric wheel-legged robot, which combined impedance control methods with the robot. The electric wheellegged robot achieves soft landing on the feet and the ground. The gait movement and mechanism of a goat on a slope were researched and the walking mechanism of the goat was designed. The results indicated that the change in gait movement during walking was mainly attained by adjusting the position of the center of gravity ${ }^{[24,25]}$. The trajectory of the head centroid movement of a goat on a slope was measured, and its influence on the stability was analyzed to solve the problem of irregular ground walking of current small machinery ${ }^{[26]}$.

As a typical mountain animal, the goat has both motion and balance control capabilities in a complex environment. They can independently choose the best landing point and carry out straddle movements. The engineering bionics technology combined with the advantages of quadruped robots and motor drives was applied. The design of a motor-driven bionic goat quadruped robot, kinematic modeling and gait planning was performed based on it, and the gait planning was also be simulated and analyzed through simulation software. An experimental platform was set up and verified by experiments to replicate the superior kinematic performance of the goat and improve quadruped robot walking stability and obstacle capability on slopes.

\section{Mechanical design}

A goat walking test platform was set up, a high-speed camera system was used to capture the image sequence of a goat walking on the slope, and the image data was processed to obtain the hip joint angle of the front leg of the goat during the walking on the slope changed from $46.1^{\circ}$ to $135.5^{\circ}$. The knee joint angle ranged from $87.9^{\circ}$ to $179.8^{\circ[27]}$. The physical characteristics of goats were obtained through experiments, as shown in Table 1. The limb parameters of the goat are shown in Table 2.

Table 1 Body characteristics of the goat

\begin{tabular}{lc}
\hline \multicolumn{1}{c}{ Parameters } & Values \\
\hline Weight $/ \mathrm{kg}$ & 18.30 \\
Total body length $/ \mathrm{m}$ & 0.86 \\
Body length $/ \mathrm{m}$ & 0.38 \\
Overall height $/ \mathrm{m}$ & 0.76 \\
Height $/ \mathrm{m}$ & 0.58 \\
\hline
\end{tabular}

Table 2 Limb parameters of the goat

\begin{tabular}{lc}
\hline \multicolumn{1}{c}{ Parameters } & Values/m \\
\hline The length of the left front thigh & 0.17 \\
The length of the left front calf & 0.19 \\
The length of the left-back thigh & 0.20 \\
The length of the left-back calf & 0.20 \\
The length of the right front thigh & 0.15 \\
The length of the right front calf & 0.21 \\
The length of the right-back thigh & 0.20 \\
The length of the right-back calf & 0.22 \\
\hline
\end{tabular}

The three-dimensional structure of the quadruped robot is shown in Figure 1. The robot is mainly composed of thighs, calves, thigh joint motors, knee joint motors, damping springs, timing belts, and bionic feet. The control instruction was input on the PC. The angle that each joint motor needed to rotate was calculated and then was sent to the motion controller through the control signal line. The motion controller sent the control signal to the servo driver, which converted it into a pulse signal and then controlled the joint motor to move every single leg to achieve the purpose of a bionic goat quadruped robot. The damping spring played a shock-absorbing role during the movement.

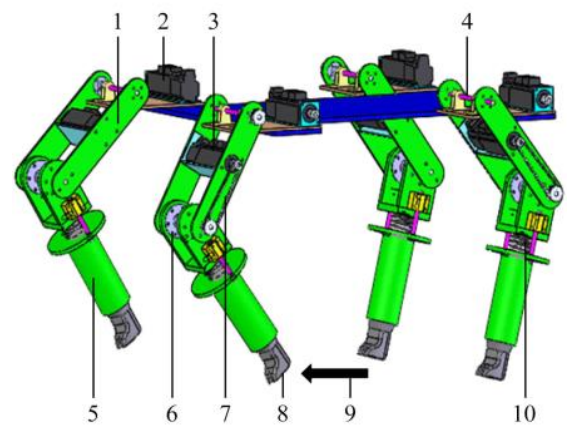

1. Thigh 2. Thigh joint motor 3. Knee joint motor 4. Hip joint 5. Calf 6. Knee joint 7. Timing belt 8. Bionic foot 9. Forward direction 10. Damping spring

Figure 1 Three-dimensional structure of the quadruped robot

The structural parameters of the quadruped robot are shown in Table 3.

Table 3 Structure parameters

\begin{tabular}{lc}
\hline Parameters & Values \\
\hline Body length $/ \mathrm{m}$ & 1.00 \\
Body width $/ \mathrm{m}$ & 0.60 \\
Body height $/ \mathrm{m}$ & 0.45 \\
Body weight $/ \mathrm{kg}$ & 25.00 \\
Thigh length $/ \mathrm{m}$ & 0.20 \\
Calf length $/ \mathrm{m}$ & 0.20 \\
\hline
\end{tabular}

\section{Kinematic analysis}

\subsection{Create Denavit-Hartenberg (D-H) coordinates}

Kinematic analysis of a quadruped robot is the key to its gait planning. By establishing a coordinate system, the posture relationship between the various components of the robot is obtained. Based on the D-H ${ }^{[28]}$ method, considering the foot position of a quadruped robot in the world coordinate system, the world coordinate system is defined as $E$, the reference coordinate system of the quadruped robot body is $B$, and its origin is located at the centroid of the quadruped robot. The base coordinate system of the left front leg is $0_{L F}$, and the base coordinate system of the right front leg is $0_{R F}$; the base coordinate system of the left hind leg is $0_{L H}$, and the base coordinate system of the right hind leg is $0_{R H}$. The coordinate system diagram is shown in Figure 2, where $m$ is half the length of the quadruped robot, and $n$ is half the width of the quadruped robot.

\subsection{Kinematics analysis}

3.2.1 Forward kinematics of the single leg

According to the D-H method, the transformation ${ }^{n} T_{n+1}$ (called $A_{n+1}$ ) between the coordinate system $n$ and the coordinate system $n+1$ is the right multiplication of the 4 motion transformation matrices.

$$
\begin{aligned}
{ }^{n} T_{n+1} & =A_{n+1} \\
& =\left[\begin{array}{cccc}
C \theta_{n+1} & -S \theta_{n+1} C \alpha_{n+1} & S \theta_{n+1} S \alpha_{n+1} & a_{n+1} C \theta_{n+1} \\
S \theta_{n+1} & C \theta_{n+1} C \alpha_{n+1} & -C \theta_{n+1} S \alpha_{n+1} & a_{n+1} S \theta_{n+1} \\
0 & S \alpha_{n+1} & C \alpha_{n+1} & d_{n+1} \\
0 & 0 & 0 & 1
\end{array}\right]
\end{aligned}
$$


where, $C=\cos , S=\sin ; \theta_{n+1}$ represents the angle of rotation around the $z_{n}$-axis; $d_{n+1}$ represents the distance of translation along the $z_{n}$-axis; $a_{n+1}$ represents the distance of translation along the $x_{n}$-axis; $\alpha_{n+1}$ represents the angle of rotating the $z_{n}$-axis around the $x_{n+1}$-axis.

Taking the right front leg as an example, the D-H method is used to establish its coordinate system, as shown in Figure 3. The definition of the coordinate system is as follows: the connection point between the body and the legs is 0 , the forward direction of the quadruped robot is the $Z$-axis, and the $X$-axis is vertically downward. The right axis rule determines that the $Y$-axis is pointing to the inside of the body.

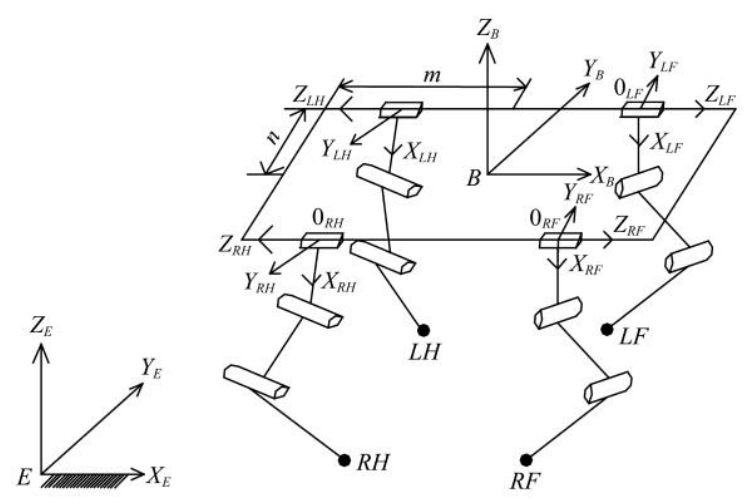

Figure 2 Relationship between the quadruped robot base coordinate system and the world coordinate system

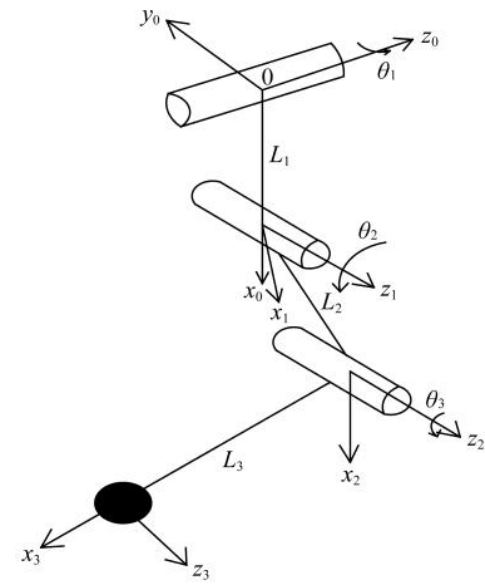

Figure 3 D-H coordinate diagram of the right front leg of the quadruped robot

The D-H parameters of the right front leg shown in Table 4 are determined, according to Figure 3.

\section{Table 4 D-H parameters table of the right front leg}

\begin{tabular}{ccccc}
\hline$\#$ & $\theta$ & $d$ & $a$ & $\alpha$ \\
\hline $0-1$ & $\theta_{1}$ & 0 & $L_{1}$ & $90^{\circ}$ \\
$1-2$ & $\theta_{2}$ & 0 & $L_{2}$ & 0 \\
$2-3$ & $\theta_{3}$ & 0 & $L_{3}$ & 0
\end{tabular}

Note: $\theta$ represents the $z$-axis rotation angle; $\theta_{1}$ represents the thigh yaw joint angle; $\theta_{2}$ represents the hip angle; $\theta_{3}$ represents the knee angle, $a$ represents the length of each common vertical line; $L_{1}$ represents the yaw joint to the hip joint length; $L_{2}$ represents the length of the thigh; $L_{3}$ represents the length of the calf; $d$ represents two adjacent vertical line spacing of the $z$-axis; $\alpha$ represents the angle between two adjacent $z$-axes.

According to Equation (1), the three D-H coordinate transformation parameters of the right front leg of the four-legged robot in Table 1 are substituted respectively, the transformation matrix of the right front leg of the quadruped robot can be obtained.

$$
\begin{aligned}
{ }^{0} T_{3} & ={ }^{0} T_{1}{ }^{1} T_{2}{ }^{2} T_{3} \\
& =A_{1} A_{2} A_{3} \\
& =\left[\begin{array}{cccc}
C_{1} C_{23} & -C_{1} S_{23} & S_{1} & C_{1}\left(L_{3} C_{23}+L_{2} C_{2}+L_{1}\right) \\
S_{1} C_{23} & -S_{1} S_{23} & -C_{1} & S_{1}\left(L_{3} C_{23}+L_{2} C_{2}+L_{1}\right) \\
S_{23} & C_{23} & 0 & L_{3} S_{23}+L_{2} S_{2} \\
0 & 0 & 0 & 1
\end{array}\right] \\
& =\left[\begin{array}{cccc}
n_{x} & o_{x} & a_{x} & p_{x} \\
n_{y} & o_{y} & a_{y} & p_{y} \\
n_{z} & o_{z} & a_{z} & p_{z} \\
0 & 0 & 0 & 1
\end{array}\right]
\end{aligned}
$$

where, $p_{x}$ represents the $x$-axis coordinate of the foot; $p_{y}$ represents the $y$-axis coordinate of the foot; $p_{z}$ represents the $z$-axis coordinate of the foot; As three unit vectors, $n, o$ and $a$ are used as the basis to form a rectangular coordinate system. nine elements in the upper left of the matrix include $n_{x}, n_{y}, n_{z}, o_{x}, o_{y}, o_{z}, a_{x}, a_{y}$ and $a_{z}$, these elements describe the posture of the end of the right front leg relative to the reference coordinate axis; $S_{1}=\sin \theta_{1} ; C_{1}=\cos \theta_{1} ; S_{2}=$ $\sin \theta_{2} ; C_{2}=\cos \theta_{2} ; S_{3}=\sin \theta_{3} ; C_{3}=\cos \theta_{3} ; S_{23}=\sin \left(\theta_{2}+\theta_{3}\right) ; C_{23}=\cos \left(\theta_{2}+\theta_{3}\right)$.

To sum up, the equation of forwarding kinematic of the right front leg of a quadruped robot is as shown in Equation (3):

$$
\left\{\begin{array}{l}
p_{x}=C_{1}\left(L_{3} C_{23}+L_{2} C_{2}+L_{1}\right) \\
p_{y}=S_{1}\left(L_{3} C_{23}+L_{2} C_{2}+L_{1}\right) \\
p_{z}=L_{3} S_{23}+L_{2} S_{2}
\end{array}\right.
$$

\subsubsection{Inverse kinematics of the single leg}

Taking the right front leg of a quadruped robot as an example, the equation of inverse kinematics was deduced. Equation (4) can be obtained from Equation (3):

$$
\theta_{1}=\arctan \frac{p_{y}}{p_{x}}
$$

The equation of inverse kinematic of the front leg of a quadruped robot can be obtained from $A_{1}^{-10} T_{3}=A_{2} A_{3}$ and $A_{2}^{-1} A_{1}^{-10} T_{3}=A_{3}$, as shown in Equation (5):

$$
\left\{\begin{aligned}
\theta_{1}= & \arctan \frac{p_{y}}{p_{x}} \\
\theta_{2}= & \arccos \frac{L_{3} S_{3}}{\sqrt{\left(C_{1} p_{x}+S_{1} p_{y}-L_{1}\right)^{2}+p_{z}^{2}}}- \\
& \arccos \frac{p_{z}}{\sqrt{\left(C_{1} p_{x}+S_{1} p_{y}-L_{1}\right)^{2}+p_{z}^{2}}} \\
\theta_{3}= & \arccos \frac{\left(C_{1} p_{x}+S_{1} p_{y}-L_{1}\right)^{2}+p_{z}^{2}-L_{2}^{2}-L_{3}^{2}}{2 L_{2} L_{3}}
\end{aligned}\right.
$$

\subsubsection{Equation of velocity and acceleration}

When the quadruped robot walking straight, the hip joint angle is zero, and $P_{y}=0$, from the equation of inverse kinematic of the front leg of a quadruped robot, Equation (6) can be obtained:

$$
\left\{\begin{array}{l}
-L_{2} L_{3} S_{3} \dot{\theta}_{3}=\left(p_{x}-L_{1}\right) \dot{p}_{x}+p_{z} \dot{p}_{z} \\
-C_{2} \dot{\theta}_{2}\left(p_{x}-L_{1}\right)-S_{2} \dot{p}_{x}+C_{2} \dot{p}_{z}-S_{2} \dot{\theta}_{2} p_{z}=L_{3} C_{3} \dot{\theta}_{3}
\end{array}\right.
$$

The velocity equation of the quadruped robot can be obtained:

$$
\left\{\begin{array}{l}
\dot{\theta}_{1}=0 \\
\dot{\theta}_{2}=-\frac{L_{3} C_{3} \dot{\theta}_{3}+S_{2} \dot{p}_{x}-C_{2} \dot{p}_{z}}{C_{2}\left(p_{x}-L_{1}\right)+S_{2} p_{z}} \\
\dot{\theta}_{3}=-\frac{\left(p_{x}-L_{1}\right) \dot{p}_{x}+p_{z} \dot{p}_{z}}{L_{2} L_{3} S_{3}}
\end{array}\right.
$$

Differentiating time $t$ on the left and right sides of Equation (6) 
at the same time, the acceleration equation of the quadruped robot

$$
\left\{\begin{array}{l}
\ddot{\theta}_{1}=0 \\
\ddot{\theta}_{2}=\frac{\left[S_{2}\left(p_{x}-L_{1}\right)-C_{2} p_{z}\right] \dot{\theta}_{2}^{2}-2\left(C_{2} \dot{p}_{x}+S_{2} \dot{p}_{z}\right) \dot{\theta}_{2}-S_{2} \ddot{p}_{x}+C_{2} \ddot{p}_{z}+L_{3} S_{3} \dot{\theta}_{3}^{2}-L_{3} C_{3} \ddot{\theta}_{3}}{C_{2}\left(p_{x}-L_{1}\right)+S_{2} p_{z}} \\
\ddot{\theta}_{3}=-\frac{L_{2} L_{3} C_{3} \dot{\theta}_{3}^{2}+\dot{p}_{x}^{2}+\left(p_{x}-L_{1}\right) \ddot{p}_{x}+\dot{p}_{z}^{2}+p_{z} \ddot{p}_{z}}{L_{2} L_{3} S_{3}}
\end{array}\right.
$$

is obtained:
Based on the analysis of the topological structure of the quadruped robot, a bionic goat quadruped robot body structure was designed. Based on the D-H method for kinematic modeling, the equations of velocity and acceleration of the quadruped robot were calculated, which provided a theoretical basis for the control of the quadruped robot.

\section{Foot-end trajectory planning of walk gait}

Gait planning is mainly divided into two parts, one is to determine the coordination relationship between the legs, that is to determine the duty ratio and phase difference, and the other is to plan a reasonable foot-end trajectory curve. The swing phase and stance phase of a quadruped robot were studied during a walking gait. In order to reduce the impact force when their feet touch the ground, a low contact compact foot trajectory planning method using high order polynomial curve is used to carry out the foot trajectory planning of robot for foot-end trajectory planning.

\subsection{Foot-end trajectory planning of swing phase}

Figure 2 shows that the quadruped robot walking direction was the $X$-direction, and the leg raising direction was the $Z$-direction. According to the requirements of the quadruped robot's foot-end movement, the displacement constraint equations of the quadruped robot's swing phase foot-end trajectory in the walking direction ( $X$-direction) and leg raising direction (Z-direction) were shown as follows:

$$
\left\{\begin{array}{c}
x_{t=0}=-\frac{S}{2} \\
x_{t=T}=\frac{S}{2}
\end{array}\right.
$$

where, $x_{t=0}$ represents the initial position; $x_{t=T}$ represents the end position; $S$ is the gait length at the foot of the swing phase, $\mathrm{cm}$; $T$ is the period of the swing phase, $s$.

$$
\left\{\begin{array}{l}
z_{t=0}=0 \\
z_{t=T / 2}=H \\
z_{t=T}=0
\end{array}\right.
$$

where, $z_{t=0}$ represents the initial position; $z_{t=T / 2}$ represents half period position; $z_{t=T}$ represents the end position; $H$ is the lifting height of the foot during the swing phase, $\mathrm{cm}$.

In order to reduce the instantaneous impact force and inertia force of the quadruped robot when the swing phase and the stance phase are switched, based on the principle of zero impact (the velocity and acceleration of the quadruped robot are zero when the foot touches the ground), the velocity and acceleration constraint equations of the foot-end trajectory curve of the quadruped robot in the walking direction ( $X$-direction) and leg raising direction (Z-direction) are shown as follows:

$$
\begin{array}{r}
\left\{\begin{array}{l}
\dot{x}_{t=0}=0 \\
\dot{x}_{t=T}=0
\end{array}\right. \\
\left\{\begin{array}{l}
\dot{z}_{t=0}=0 \\
\dot{z}_{t=T}=0
\end{array}\right.
\end{array}
$$

$$
\begin{array}{r}
\left\{\begin{array}{l}
\ddot{x}_{t=0}=0 \\
\ddot{x}_{t=T}=0
\end{array}\right. \\
\left\{\begin{array}{l}
\ddot{z}_{t=0}=0 \\
\ddot{z}_{t=T}=0
\end{array}\right.
\end{array}
$$

where, $\dot{x}_{t=0}$ represents the initial velocity; $\dot{x}_{t=T}$ represents the end velocity; $\dot{z}_{t=0}$ represents the initial velocity; $\dot{z}_{t=T}$ represents the end velocity; $\ddot{x}_{t=0}$ represents the initial acceleration; $\ddot{x}_{t=T}$ represents the end acceleration; $\ddot{z}_{t=0}$ represents the initial acceleration; $\ddot{z}_{t=T}$ represents the end acceleration.

A low contact compact foot trajectory planning method using high order polynomial curve was used to carry out the foot trajectory planning of the robot for foot-end trajectory planning, and the equation of the foot trajectory was deduced by a polynomial. From Equations (9), (11), and (13), the foot-end trajectory has six constraints in the walking direction ( $X$ direction), and the foot-end trajectory in the walking direction $x$ is a quintic polynomial:

$$
x=A t^{5}+B t^{4}+C t^{3}+D t^{2}+E t+F
$$

where, $\mathrm{A}$ is the coefficient of $t^{5}$; B is the coefficient of $t^{4} ; \mathrm{C}$ is the coefficient of $t^{3}$; D is the coefficient of $t^{2} \mathrm{E}$ is the coefficient of $t ; \mathrm{F}$ is a constant.

Substituting Equations (9), (11), and (13) into Equation (15), the equation of the foot-end trajectory curve of the quadruped robot can be calculated as follows:

$$
x=\frac{6 S}{T^{5}} t^{5}-\frac{15 S}{T^{4}} t^{4}+\frac{10 S}{T^{3}} t^{3}-\frac{S}{2}
$$

Similarly, the trajectory curve equation of the foot-end trajectory of the quadruped robot in the direction of raising the leg $z$ is as follows:

$$
z=-\frac{64 H}{T^{6}} t^{6}+\frac{192 H}{T^{5}} t^{5}-\frac{192 H}{T^{4}} t^{4}+\frac{64 H}{T^{3}} t^{3}
$$

\subsection{Foot-end trajectory planning of stance phase}

When changing the phase of the support leg, the trunk of the quadruped robot is moved in the horizontal direction. In the planning of the stance phase, it is assumed that the sole of the foot and the contact surface do not slide relative to each other, and the trunk is parallel to the contact surface. The body moves forward under the push of the support leg, which is equivalent to moving in the opposite direction. A low contact compact foot trajectory planning method using a high order polynomial curve was used to the planning of the stance phase, the constraints satisfied the Equation (18):

$$
\left\{\begin{array}{l}
x_{t=0}=\frac{S}{2} \\
x_{t=T}=-\frac{S}{2} \\
\dot{x}_{t=0}=0 \\
\dot{x}_{t=T}=0 \\
\ddot{x}_{t=0}=0 \\
\ddot{x}_{t=T}=0
\end{array}\right.
$$


The foot-end stance phase trajectory of a quadruped robot satisfied the constraint condition of Equation (18), and the foot-end trajectory equation of the stance phase was set to a quintic polynomial:

$$
x=A t^{5}+B t^{4}+C t^{3}+D t^{2}+E t+F
$$

Similarly, by substituting Equation (18) into Equation (19), the foot-end trajectory curve equation of the stance phase of the quadruped robot can be calculated as follows:

$$
x=-\frac{6 S}{T^{5}} t^{5}+\frac{15 S}{T^{4}} t^{4}-\frac{10 S}{T^{3}} t^{3}+\frac{S}{2}
$$

\subsection{Foot trajectory curve of walking gait}

According to the previous goat slope walking test, the average gait length $S=30 \mathrm{~cm}$, average step height $H=7 \mathrm{~cm}$, and average gait period $T=4 \mathrm{~s}$ were obtained when the goat was walking. Based on the curve equation of the foot-end trajectory of the quadruped robot, the relationship between the displacement and time, velocity and time, and acceleration and time of the swing phase in the walking direction and the leg raising direction were obtained using MATLAB simulation and shown in Figure 4.

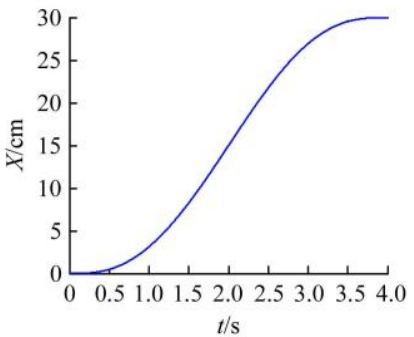

$X$-direction

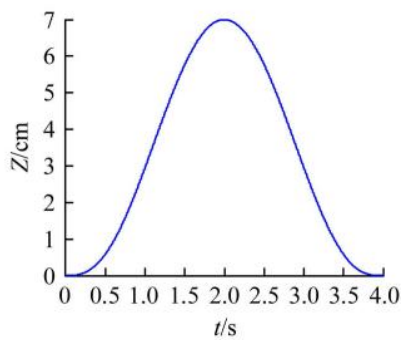

Z-direction

a. Variation of the displacement of the swing phase in different directions with time

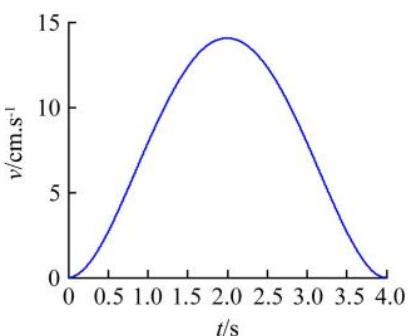

$X$-direction

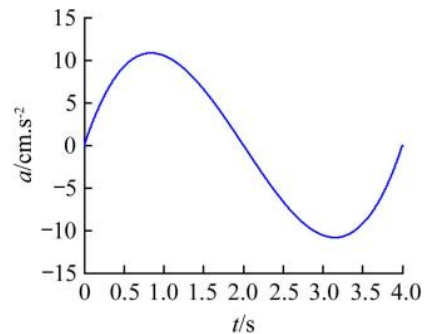

$X$-direction

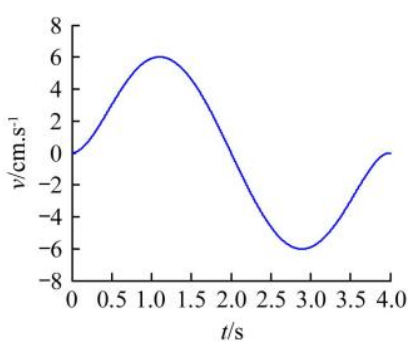

Z-direction

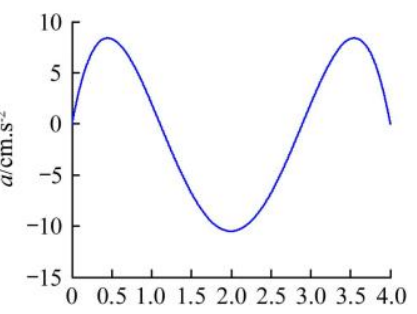

$t / \mathrm{s}$

$$
\text { Z-direction }
$$

c. Graph of acceleration versus time in different directions

Figure 4 Graph of the swing phase foot-end movement of a quadruped robot

The simulation results of Figure 4 show that the foot-end trajectory planning of the walking gait of the quadruped robot is reasonable, and the goal of zero impact can be achieved.

The change curve of the foot-end trajectory of the quadruped robot with time is obtained using MATLAB simulation, as shown in Figure 5.

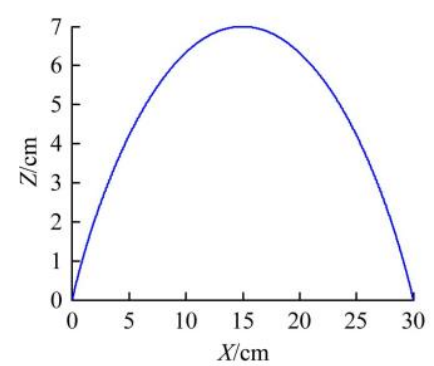

a. Curve of the foot trajectory of the swing phase in the $\mathrm{XOZ}$ plane

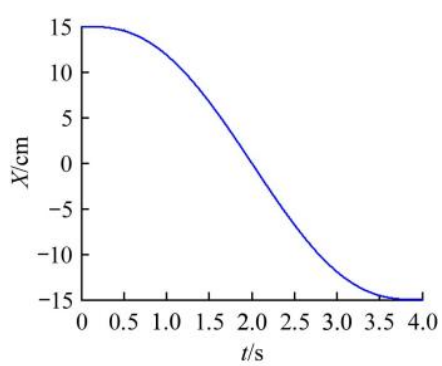

b. Foot motion curve of the stance phase
Figure 5 Graph of the foot-end trajectory of a quadruped robot

The goat slope walking test was completed earlier to obtain the kinematic parameters of the goat walking on different slopes. The data of a complete gait cycle was analyzed, and the foot trajectory of the goat walking on the slope was obtained. The goat's foot trajectory was semi-elliptical ${ }^{[24]}$. In a gait cycle, the foot-end trajectory of the quadruped robot swing phase in the $\mathrm{XOZ}$ plane was semi-elliptical, as shown in Figure 5, which was consistent with the results of the foot-end trajectory when walking on a sloping hill. It further verified the rationality of foot-end trajectory planning for the walking gait of a quadruped robot.

\subsection{Gait planning of walking gait}

As a typical static gait, the walking gait should meet the requirements of static stability. When a quadruped robot walks in a static gait, 3 legs are in the stance phase, and the centroid of the body falls inside the triangle connected by the stance phase projection point ${ }^{[29]}$. The theory of stability margin is used to measure the static stability of a quadruped robot. The stability margin represents the shortest distance from the projection point to the 3 sides, as shown in Equation (21).

$$
\lambda=\min \left(D_{1}, D_{2}, D_{3}\right)
$$

where, $\lambda$ represents for stability margin; $D_{1}, D_{2}, D_{3}$ represents the shortest distance from the vertical projection point of the robot's centroid to the three sides in the triangle.

When the quadruped robot is in a walking gait, the stability margin should satisfy the condition of $\lambda \geq 0$, and the larger the $\lambda$ value is, the better the static stability of the quadruped robot is.
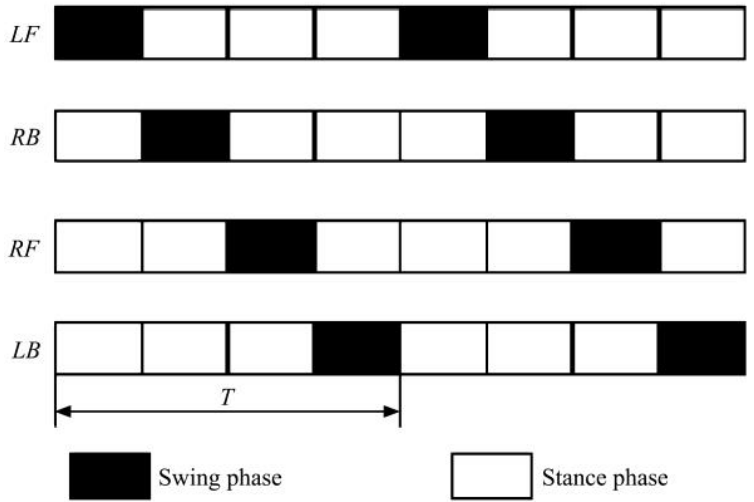

Figure 6 Diagram of walk gait of a quadruped robot

The quadruped robot has a duty ratio of $0.75<\beta<1$ when walking in static gait. The sequence of leg-lifting step $L F-R B-R F-L B$ is the best choice for the stability margin. Therefore, using the sequence of leg-raising step and duty ratio $\beta=0.75$ to plan the walking gait, the schematic diagram of the walking gait is obtained and shown in Figure 6, where, $L F$ is the left front leg, $R F$ is the right front leg, $R B$ is the right-back leg, and $L B$ is the left back leg. 


\section{Construction and test of the whole machine platform}

\subsection{Construction of the whole machine platform}

The test platform of the bionic quadruped robot was built. As shown in Figure 7, the test platform includes a bionic quadruped robot test model, controller, motor and motor driver. The physical model of a bionic quadruped robot is a series of 4 legs exactly the same, which are installed on the body of the quadruped robot in the manner of front knee and elbow. A motion controller (GUC T Series, Googol Technology, HongKong, China) was adopted. 8 servo drives (ASD-A2-0121-E, company, address, country) were used in the actuator. Two kinds of servo motors (ECMA-C10401HS by 4, ECMA-C10604SS by 4, company, address, Country) were used. The power system includes $24 \mathrm{~V}$ power of motor brake and controller power.

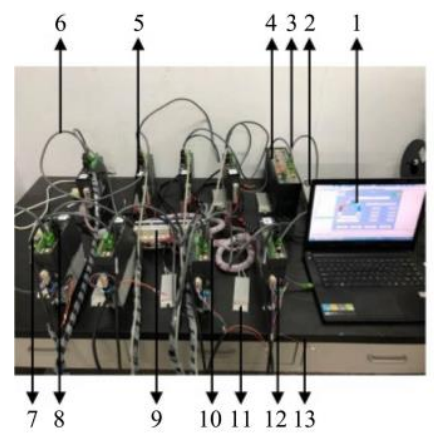

a. Control system

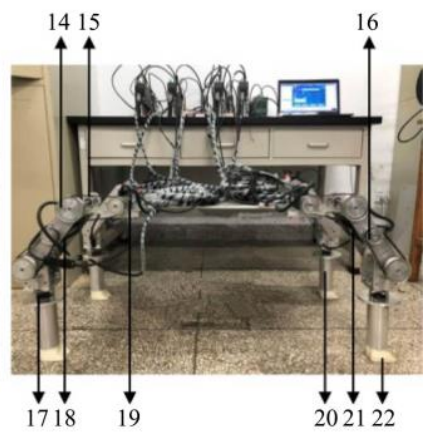

b. Mechanical structure

1. Computer 2. Controller power supply 3 . Control signal line $\quad 4$. Controller 5. Driver 6. Super five shielded signal line 7. Brake power supply 8. Encoder line 9. Control switch 10. Driver power line 11. Regeneration resistor 12. Motor power line 13. Motor brake line 14. Reduction wheel 15. Timing belt 16 . Synchronous wheel 17 . Damping spring 18 . Knee joint motor 19. Thigh joint motor 20. Calf 21 . Thigh 22. Bionic foot

Figure 7 Test platform of the bionic goat quadruped robot

\subsection{Test of whole machine platform}

This test was planned according to the walking gait, with $S=$ $30 \mathrm{~cm}$ and $H=7 \mathrm{~cm}$. By controlling the servo motor driver of each

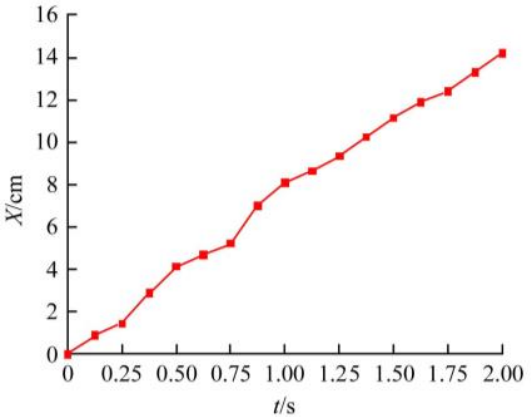

a. Movement curve of displacement of $X$-direction with time

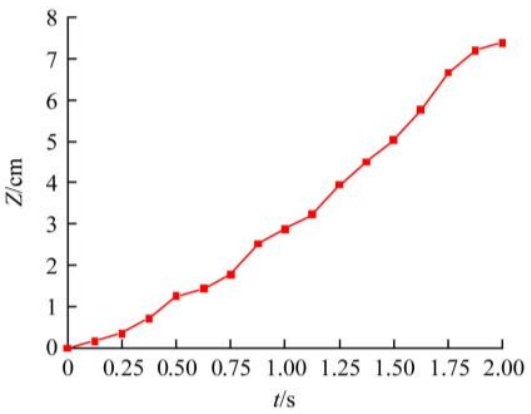

d. Movement curve of displacement of $Z$-direction with time

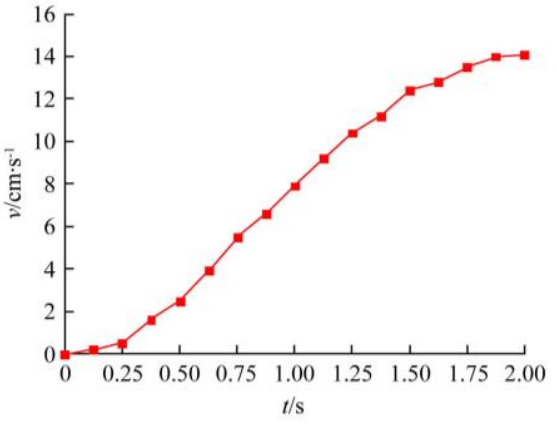

b. Motion curve of the velocity of $X$-direction with time

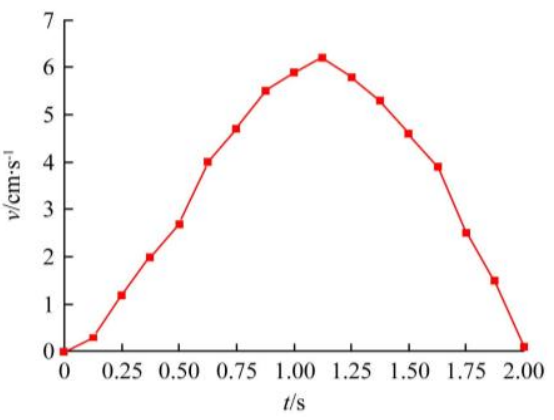

e. Movement curve of the velocity of $Z$-direction with time leg joint, 4 legs of the quadruped robot move according to the planned gait sequence. In order to verify the consistency of the motion parameters of the quadruped robot with the planning parameters, the high-speed camera technology was used to capture the motion state of the quadruped robot to obtain a sequence of steps for the quadruped robot to raise legs. In order to analyze the foot-end trajectory of a quadruped robot as it walked up the legs, the image sequence was captured by the high-speed camera test and processed. The upper right corner of the image was set as the origin of the coordinates, and the measurement method was Distance\&Angle\&Speed: Origin +1 point. The foot position of the right front leg of the quadruped robot was measured at a 200 -frame interval, as shown in Figure 8

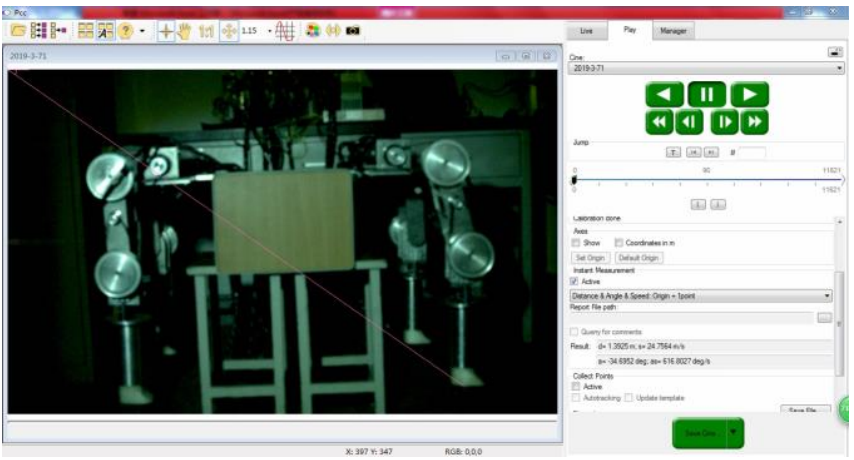

Figure 8 Test processing

By analyzing the high-speed camera results, the motion parameters of the quadruped robot in half a cycle were obtained, as shown in Figure 9.

From Figure 9, the leg-lifting height of the quadruped robot is $7.37 \mathrm{~cm}$, and the gait length is $28.4 \mathrm{~cm}$. Compared with the planned $H=7 \mathrm{~cm}$ and $S=30 \mathrm{~cm}$, the errors of the leg-lifting height and the gait length is $5.28 \%$ and $5.33 \%$. The velocity and acceleration of the quadruped robot when moving in the forward direction ( $X$-direction) and leg raising direction (Z-direction) are in good agreement with the planned velocity and acceleration. Therefore, the results of the designed gait plan achieve the desired effect. 


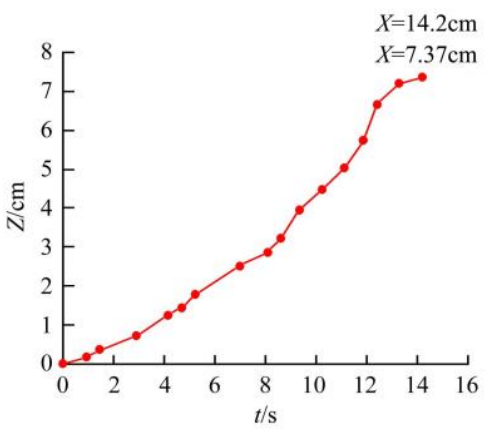

g. Foot movement curve of the quadruped robot in the $\mathrm{XOZ}$ plane

Figure 9 Motion parameters of the quadruped robot

The motion image sequence of the quadruped robot was analyzed, and the angles of the knee and hip corresponding to each moment in its half-cycle were obtained, as shown in Table 5.

Table 5 Changes of joint Angle of the quadruped robot

\begin{tabular}{ccc}
\hline Time/s & Joint angle $/\left(^{\circ}\right)$ & Hip joint angle $/\left(^{\circ}\right)$ \\
\hline 0 & 156.1 & 103.9 \\
0.125 & 155.9 & 104.7 \\
0.250 & 154.6 & 108.0 \\
0.375 & 149.6 & 108.9 \\
0.500 & 151.5 & 112.3 \\
0.625 & 147.4 & 112.4 \\
0.750 & 146.9 & 115.7 \\
0.875 & 145.7 & 117.6 \\
1.000 & 136.9 & 117.9 \\
1.125 & 140.6 & 123.5 \\
1.250 & 135.5 & 121.9 \\
1.375 & 134.4 & 128.8 \\
1.500 & 129.5 & 133.9 \\
1.625 & 128.8 & 129.5 \\
1.750 & 129.3 & 132.5 \\
1.875 & 126.3 & 138.4 \\
2.000 & 129.5 & 137.7 \\
\hline & &
\end{tabular}

As shown in Table 5, the knee angle of the quadruped robot varies from $126.3^{\circ}$ to $156.1^{\circ}$, hip joint angles range from $103.9^{\circ}$ to $138.4^{\circ}$. To compare with the angle of the knee joint ranging from $87.9^{\circ}$ to $179.8^{\circ}$ and hip joint angle changing from $46.1^{\circ}$ to $170.0^{\circ}$ when the goat walking on the slope, the range of the angle of the knee and hip joints of the planned quadruped robot is within the range of the actual angle of the goat as it walks.

\section{Conclusions}

In this research, the mechanical body of the quadruped robot driven by the motor was designed, and the kinematic analysis was performed to obtain the velocity and acceleration equations of the joint angle changing during its movement. It provided a theoretical reference for the establishment of the control system of the quadruped robot in the next step.

The foot trajectory of the static gait was planned and then simulated using MATLAB software. The simulation results showed the rationality of the foot-end trajectory planning. Then gait planning was performed to obtain a sequence diagram of the leg-up step of a quadruped robot during a walking gait.

The bionic quadruped robot test platform was built and verified by experiments. The results showed that the error between the leg height and the gait length in the walk state was $5.28 \%$ and $5.33 \%$, and the gait planning achieved the expected effect. The knee angle and hip angle of the quadruped robot were within the range of actual changes when the goat walked. The rationality of the mechanism design was proved, and the feasibility of establishing mathematical models and gait planning was verified. It provided a reference for the subsequent research of quadruped robots walking on slopes.

\section{Acknowledgements}

The authors acknowledge that this research was supported by the National Key Research and Development Program of China project (Grant No. 2017YFD0301106), the National Natural Science Foundation of China (Grant No. 52075149, 51675163); the Open Project Program of Key Laboratory of Modern Agricultural Equipment and Technology, Ministry of Education and High-tech Key Laboratory of Agricultural Equipment \& Intelligentization of Jiangsu Province (Grant No. JNZ201901); the Colleges and Universities of Henan Province Youth Backbone Teacher Training Program (Grant No. 2017GGJS062); the Postgraduate Education Reform Project of Henan Province (Grant No. 2019SJGLX063Y).

\section{[References]}

[1] Wang C W, Ma K, Yang L, Ma H W, Xue X S, Tian H B. Simulation and experiment on obstacle-surmounting performance of four swing arms and six tracked robot under unilateral step environment. Transaction of the CSAE, 2018; 34(10): 46-53. (in Chinese)

[2] Kumar A, Ojha A. Experimental evaluation of certain pursuit and evasion schemes for wheeled mobile robots. International Journal of Automation and Computing, 2019; 16(4): 491-510.

[3] Zhang C, Yang M J, Chen J, Jiang M, Ma Y C, Ji J. Energy consumption optimization model of agricultural hexapod robot with self-locking joints. Transaction of the CSAE, 2016; 32(18): 73-83. (in Chinese)

[4] Wang X L, Jin Z L, Li X D. Analysis and verification of terminal position error of leg parallel mechanism of quadruped robot. Transaction of the CSAE, 2016; 32(18): 73-83. (in Chinese)

[5] Shan L J, Yu Z H, Dai Y X, Zhang D Z. Research on intelligent inspection robotic system for electric vehicle charging station. Zhejiang Electric Power, 2018; 37(6): 47-52. (in Chinese)

[6] Huang Q, Kazuhito Y, Shuuji K, Kenji K, Hirohiko A, Noriho K, et al. Planning walking patterns for a biped robot. Institute of Electrical and Electronics Engineers, 2001; 17(3): 280-289.

[7] Zhang S S, Rong X W, Li Y B, Li B. Static gait planning method fo quadruped robots on rough terrains. Journal of Jilin University (Engineering and Technology Edition), 2016; 46(4): 1287-1296. (in Chinese)

[8] Yin Y H, Bian X G, Lyu W L. Gait planning and motion control on the hydraulic pressure actuated quadruped robot. Journal of Mechanical \& Electrical Engineering, 2014; 31(7): 839-843. (in Chinese)

[9] Faigl J, Cížek P. Adaptive locomotion control of hexapod walking robot for traversing rough terrains with position feedback only. Robotics and Autonomous Systems, 2019;116: 136-147.

[10] Liu Y W, Liu S W, Mei T, Wu X, Li Y. Design and analysis of a bio-inspired tracked wall-climbing robot with spines. Robot, 2019; 41(4): 526-533. (in Chinese)

[11] Tanveer M H, Recchiuto C T, Sgorbissa A. Analysis of path following 
and obstacle avoidance for multiple wheeled robots in a shared workspace. Cambridge University Press, 2018; 37(1): 1-29.

[12] Zhu Y, Wang M H, Li B, Wang C. Mechanism-parameters design and validation of transformable tracked robot based on goal programming. Transaction of the CSAE, 2016; 32(14): 39-46. (in Chinese)

[13] Zhang J, Chen D, Wang S M, Hu X A, Wang D. Design and experiment of four-wheel independent steering driving and control system for agricultural wheeled robot. Transaction of the CSAE, 2015; 31(18): 63-70. (in Chinese)

[14] Zhang J Z, Jin Z L, Zhang T H. Kinematic/static performance evaluation and geometric parameter design of parallel-driving leg mechanism. Transaction of the CSAE, 2017; 33(21): 61-69. (in Chinese)

[15] Tian X H, Gao F, Chen X B, Qi C K. Mechanism design and comparison for quadruped robot with parallel-serial leg. Journal of Mechanical Engineering, 2013; 49(6): 81-88. (in Chinese)

[16] Ma Z L, Zhang P Q, Lü R J, Wang J M. Stability analysis of walking on the slope for a quadruped robot. Journal of Northeastern University (Natural Science), 2018; 39(5): 673-678. (in Chinese)

[17] Lu K X, Qin J J. Design of hydraulic four-legged robot under the demand of non-structural environment. Jourmal of Chongqing University of Technology (Natural Science), 2019; 33(9): 132-141. (in Chinese)

[18] Chang Q, Han B L, Qiao Z X, Li X. An adaptive control algorithm for quadruped robot trotting on a slope. Transactions of Beijing Institute of Technology, 2019; 39(9): 900-906. (in Chinese)

[19] Yang J J, Sun H, Wang C H, Chen X D. An overview of quadruped robots. Navigation Positioning \&Timing, 2019; 6(5): 61-73. (in Chinese)

[20] Hodoshima R, Doi T, Fukuda Y, Hirose S, Okamoto T, Mori J. Development of TITAN XI: a quadruped walking robot to work on slopes. 2004 IEEE/RSJ International Conference on Intelligent Robots and Systems (IROS), Sendai: IEEE, 2004; 1: 792-797.
[21] Gao B W, Han W L. Neural network model reference decoupling control for single leg joint of hydraulic quadruped robot. Assembly Automation, 2018; 38(4): 465-475.

[22] Jiao J, Chen J, Qiao Y, Wang W Z, Wang M S, Gu L C, et al. Adaptive sliding mode control of trajectory tracking based on DC motor drive for agricultural tracked robot. Transaction of the CSAE, 2018; 34(4): 64-70. (in Chinese)

[23] Zhao J B, Chen Y H, Wang J Z. Research on leg impedance control for electronic wheel-legged robot based on fractional order. Transactions of Beijing Institute of Technology, 2019; 39(2): 187-192. (in Chinese)

[24] Zhang F, Zheng L M, Wang W, Wang Y F, Wang J J. Development of agricultural bionic mechanisms: Investigation of the effects of joint angle and pressure on the stability of goats moving on sloping lands. Int J Agric \& Biol Eng, 2018; 11(3): 35-41.

[25] Zhang F, Wang W, Zhang G Y, Wang J, Qiu Z M, Mao P J. Gait analysis of goat at different slopes and study on biomimetic walking mechanism. Int J Agric \& Biol Eng, 2016; 9(3): 40-47.

[26] Zhang F, Wang Y F, Teng S, Zheng L M, Wang J J, Chen Z J. Development of agricultural bionic four-legged robot: Effect of head movement adjustment on the stability of goats. Int J Agric \& Biol Eng, 2019; 12(4): 10-15.

[27] Zhang F, Wang Y F, Zheng L M, Ma T L, Wang J J. Biomimetic walking mechanisms: Kinematic parameters of goats walking on different slopes. Concurrency and Computation Practice and Experience, 2018; 30(24): $1-10$.

[28] Craig J J. Introduction to robotics (fourth edition). Beijing: China Machine Press, 2018.

[29] Hao R J, Wang J Z, Shi D W, Wang S K. Intermittent gait planning method of quadruped robot based on velocity vector. Robot, 2016; 38(5): 540-549. (in Chinese) 